\title{
Erratum
}

\section{Insights from Single-File Diffusion into Cooperativity in Higher Dimensions}

\author{
[Biophysical Reviews and Letters, Vol. 11, No. 1 (2016) 9-38] \\ Takeshi Ooshida \\ Department of Mechanical and Aerospace Engineering \\ Tottori University, Tottori 680-8552, Japan \\ ooshida@damp.tottori-u.ac.jp \\ Susumu Goto \\ Graduate School of Engineering Science \\ Osaka University, Toyonaka, Osaka 560-8531, Japan \\ Takeshi Matsumoto \\ Division of Physics and Astronomy \\ Graduate School of Science, Kyoto University \\ Kyoto 606-8502, Japan \\ Michio Otsuki \\ Department of Materials Science \\ Shimane University, Matsue 690-8504, Japan
}

Accepted 21 August 2017

Published 7 September 2017

There was a typographical error in Eq. (64b) of the original paper.

This was the error in the original paper:

$$
\psi_{\mathrm{r}}(\mathbf{k}, t)=\frac{k_{1}}{k_{2}} \check{\Psi}_{1}(\mathbf{k}, t)-\frac{k_{2}}{k_{1}} \check{\Psi}_{2}(\mathbf{k}, t),
$$

The error is corrected below:

$$
\psi_{\mathrm{r}}(\mathbf{k}, t)=\frac{k_{1}}{k_{2}} \check{\Psi}_{2}(\mathbf{k}, t)-\frac{k_{2}}{k_{1}} \check{\Psi}_{1}(\mathbf{k}, t),
$$

